Acta Crystallographica Section E

Structure Reports

Online

ISSN 1600-5368

\section{Ethyl 8-(2,4-dichlorophenyl)-6-methyl- 1,2,4-triazolo[1,5-a]pyridine-7-carboxyl- ate}

\section{Yang Li,* Chen Sun and Ran Zhang}

School of Chemical Engineering, Taishan Medical University, Taian 271016 People's Republic of China

Correspondence e-mail: chemyangli@gmail.com

Received 29 October 2013; accepted 6 November 2013

Key indicators: single-crystal X-ray study; $T=298 \mathrm{~K}$; mean $\sigma(\mathrm{C}-\mathrm{C})=0.004 \AA$; $R$ factor $=0.055 ; w R$ factor $=0.170 ;$ data-to-parameter ratio $=13.6$.

In the title compound, $\mathrm{C}_{16} \mathrm{H}_{13} \mathrm{Cl}_{2} \mathrm{~N}_{3} \mathrm{O}_{2}$, the carboxylate group and the benzene ring attached to the central 1,2,4-triazolo[1,5-a]pyridine bicycle are twisted from its mean plane by $55.6(1)$ and $72.6(1)^{\circ}$, respectively. In the crystal, weak $\mathrm{C}-$ $\mathrm{H}$... O interactions link the molecules into zigzag chains propagating in [100].

\section{Related literature}

For applications of $[1,2,4]$ triazolo[1,5-a]pyridine derivatives, see: Luo \& Hu (2006); Liu \& Hu (2002). For details of the synthesis, see: Jones \& Sliskovic (1983); Wang et al. (2003); Ge et al. (2009); Jia et al. (2010). For standard bond lengths, see: Allen et al. (1987).<smiles>CCOC(=O)c1c(C)cn2ncnc2c1-c1ccc(Cl)cc1Cl</smiles>

\section{Experimental}

Crystal data

$\mathrm{C}_{16} \mathrm{H}_{13} \mathrm{Cl}_{2} \mathrm{~N}_{3} \mathrm{O}_{2}$

$M_{r}=350.19$

Orthorhombic, $\mathrm{Pbca}$

$a=14.693$ (2) ̊

$b=13.531(2) \AA$

$c=16.347$ (2) §

$V=3250.0(8) \AA^{3}$

$Z=8$

Mo $K \alpha$ radiation

$\mu=0.41 \mathrm{~mm}^{-1}$

$T=298 \mathrm{~K}$

$0.33 \times 0.26 \times 0.21 \mathrm{~mm}$

Data collection

Brucker SMART APEXII CCD area-detector diffractometer Absorption correction: multi-scan (SADABS; Bruker, 1999) $T_{\text {min }}=0.876, T_{\max }=0.919$

15766 measured reflections 2860 independent reflections 2206 reflections with $I>2 \sigma(I)$

$R_{\text {int }}=0.090$

Refinement

$R\left[F^{2}>2 \sigma\left(F^{2}\right)\right]=0.055$

$w R\left(F^{2}\right)=0.170$

$S=1.07$

2860 reflections

210 parameters

$\mathrm{H}$-atom parameters constrained

$\Delta \rho_{\max }=0.42{\mathrm{e} \AA^{-3}}^{-3}$

$\Delta \rho_{\min }=-0.33 \mathrm{e}^{-3}$

Table 1

Hydrogen-bond geometry $\left(\AA{ }^{\circ}\right)$.

\begin{tabular}{lllll}
\hline$D-\mathrm{H} \cdots A$ & $D-\mathrm{H}$ & $\mathrm{H} \cdots A$ & $D \cdots A$ & $D-\mathrm{H} \cdots A$ \\
\hline $\mathrm{C} 7-\mathrm{H} 7 \cdots \mathrm{O}^{\mathrm{i}}$ & 0.93 & 2.55 & $3.296(4)$ & 137 \\
\hline
\end{tabular}

Symmetry code: (i) $x+\frac{1}{2}, y,-z+\frac{1}{2}$.

Data collection: SMART (Bruker, 1998); cell refinement: SAINT (Bruker, 1999); data reduction: SAINT; program(s) used to solve structure: SHELXS97 (Sheldrick, 2008); program(s) used to refine structure: SHELXL97 (Sheldrick, 2008); molecular graphics: SHELXTL (Sheldrick, 2008); software used to prepare material for publication: SHELXTL.

Supplementary data and figures for this paper are available from the IUCr electronic archives (Reference: CV5435).

\title{
References
}

Allen, F. H., Kennard, O., Watson, D. G., Brammer, L., Orpen, A. G. \& Taylor, R. (1987). J. Chem. Soc. Perkin Trans. 2, pp. S1-19.

Bruker (1998). SMART. Bruker AXS Inc., Madison, Wisconsin, USA.

Bruker (1999). SADABS and SAINT. Bruker AXS Inc., Madison, Wisconsin, USA.

Ge, Y. Q., Jia, J., Yang, H., Zhao, G. L., Zhan, F. X. \& Wang, J. W. (2009). Heterocycles, 78, 725-736.

Jia, J., Ge, Y. Q., Tao, X. T. \& Wang, J. W. (2010). Heterocycles, 81, 185-194. Jones, G. \& Sliskovic, D. R. (1983). Adv. Heterocycl. Chem. 34, 79-143.

Liu, T. \& Hu, Y. (2002). Bioorg. Med. Chem. Lett. 12, 2411-2413.

Luo, Y. \& Hu, Y. (2006). Arch. Pharm. Chem. Life Sci. 339, 262-266.

Sheldrick, G. M. (2008). Acta Cryst. A64, 112-122.

Wang, J. W., Jia, J., Hou, D. J., li, H. M. \& Yin, J. (2003). Chin. J. Org. Chem. 23, 173-175. 


\section{supporting information}

Acta Cryst. (2013). E69, o1796 [doi:10.1107/S160053681303033X]

\section{Ethyl 8-(2,4-dichlorophenyl)-6-methyl-1,2,4-triazolo[1,5-a]pyridine-7-carboxyl- ate}

\section{Yang Li, Chen Sun and Ran Zhang}

\section{S1. Comment}

The $[1,2,4]$ triazolo[1,5-a]pyridine derivatives exhibit antifungal, anticancer and anti-inflammatory activities (Liu \& Hu, 2002; Luo \& $\mathrm{Hu}, 2006)$. However, only small number of $[1,2,4]$ triazolo[1,5-a]pyridines is known. The commonly used synthetic methods are the annulation of 1,2,4-triazole ring starting with amino substituted pyridines by a multistep procedure (Jones \& Sliskovic, 1983). Recently, imidazo[1,5- $a$ ]pyridines, pyrazolo[1,5- $a$ ]pyridines, imidazo[1,2a]pyridines and indolizines have been synthesized in our group wityh the use of a novel tandem reaction (Wang et al., 2003; Ge et al., 2009; Jia et al., 2010). We tried to extend this reaction to synthesize the $[1,2,4]$ triazolo[1,5-a]pyridine heterocycles and obtained the title compound (I). Herewith we present its crystal structure.

In (I) (Fig. 1), all bond lengths and angles are normal (Allen et al., 1987). The carboxylate group and benzene ring attached to the central $[1,2,4]$ triazolo[ $[1,5-a]$ pyridine bicycle are twisted from its mean plane at $55.6(1)$ and $72.6(1)^{\circ}$, respectively. In the crystal, weak intermolecular $\mathrm{C}-\mathrm{H} \cdots \mathrm{O}$ interactions (Table 1) link molecules into zigzag chains propagated in [100].

\section{S2. Experimental}

(2,4-Dichlorophenyl)(1H-1,2,4-triazol-5-yl)methanone (6 mmol), ethyl 4-bromo-3-methylbut-2-enoate (12 mmol), potassium carbonate $(1.8 \mathrm{~g}, 13.2 \mathrm{mmol})$ and DMF $(30 \mathrm{ml})$ were added to a $100 \mathrm{ml}$ round-bottomed flask. The reaction system was stirred for $8 \mathrm{~h}$. Then the mixture was poured into water $(200 \mathrm{ml})$ and extracted with dichloromethane $(3 \times 50$ $\mathrm{ml}$ ). Organic layers were combined and dried over anhydrous $\mathrm{Na}_{2} \mathrm{SO}_{4}$, then filtered. By rotary evaporation, the mixture was concentrated. After that, these crude products were depurated by using column chromatography in $76 \%$ isolated yield. Crystals suitable for X-ray diffraction analysis were obtained by slow evaporation of a solution of the title compound in a hexane/ethyl acetate mixture $(3: 1 \mathrm{v} / \mathrm{v})$ at room temperature over a period of one week.

\section{S3. Refinement}

All $\mathrm{H}$ atoms were found on difference maps, but placed in idealized positions $(\mathrm{C}-\mathrm{H}=0.93-0.97 \AA)$, and included in the final cycles of refinement using a riding model, with $U_{\mathrm{iso}}(\mathrm{H})=1.2 U_{\mathrm{eq}}(\mathrm{C})$ and $1.5 U_{\mathrm{eq}}(\mathrm{C})$ for the methyl $\mathrm{H}$ atoms. 


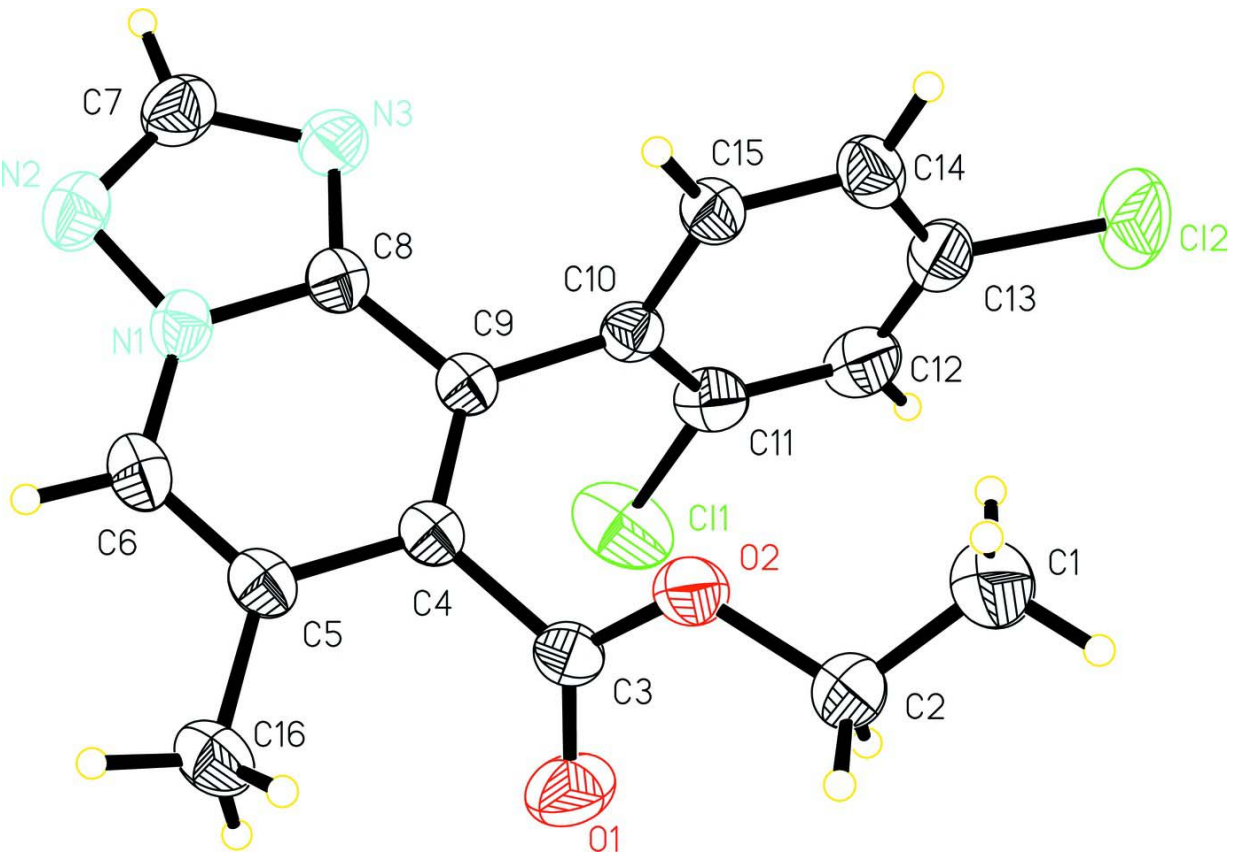

Figure 1

View of (I) with displacement ellipsoids drawn at the $30 \%$ probability level.

Ethyl 8-(2,4-dichlorophenyl)-6-methyl-1,2,4-triazolo[1,5-a]pyridine-7-carboxylate

Crystal data

$\mathrm{C}_{16} \mathrm{H}_{13} \mathrm{Cl}_{2} \mathrm{~N}_{3} \mathrm{O}_{2}$

$M_{r}=350.19$

Orthorhombic, $\mathrm{Pbca}$

Hall symbol: -P $2 \mathrm{ac} 2 \mathrm{ab}$

$a=14.693(2) \AA$

$b=13.531(2) \AA$

$c=16.347(2) \AA$

$V=3250.0(8) \AA^{3}$

$Z=8$

$F(000)=1440$

$D_{\mathrm{x}}=1.431 \mathrm{Mg} \mathrm{m}^{-3}$

Mo $K \alpha$ radiation, $\lambda=0.71073 \AA$

Cell parameters from 5439 reflections

$\theta=2.4-26.6^{\circ}$

$\mu=0.41 \mathrm{~mm}^{-1}$

$T=298 \mathrm{~K}$

Block, colourless

$0.33 \times 0.26 \times 0.21 \mathrm{~mm}$

Data collection

Brucker SMART APEXII CCD area-detector diffractometer

Radiation source: fine-focus sealed tube

Graphite monochromator

phi and $\omega$ scans

Absorption correction: multi-scan

(SADABS; Bruker, 1999)

$T_{\min }=0.876, T_{\max }=0.919$

15766 measured reflections

2860 independent reflections

2206 reflections with $I>2 \sigma(I)$

$R_{\text {int }}=0.090$

$\theta_{\text {max }}=25.0^{\circ}, \theta_{\text {min }}=2.4^{\circ}$

$h=-15 \rightarrow 17$

$k=-14 \rightarrow 16$

$l=-19 \rightarrow 19$

Refinement

Refinement on $F^{2}$

Least-squares matrix: full

$R\left[F^{2}>2 \sigma\left(F^{2}\right)\right]=0.055$

$w R\left(F^{2}\right)=0.170$

$S=1.07$

2860 reflections

210 parameters

0 restraints

Primary atom site location: structure-invariant direct methods 
Secondary atom site location: difference Fourier map

Hydrogen site location: inferred from neighbouring sites

$\mathrm{H}$-atom parameters constrained

$$
\begin{aligned}
& w=1 /\left[\sigma^{2}\left(F_{\mathrm{o}}{ }^{2}\right)+(0.081 P)^{2}+1.792 P\right] \\
& \text { where } P=\left(F_{\mathrm{o}}{ }^{2}+2 F_{\mathrm{c}}{ }^{2}\right) / 3 \\
& (\Delta / \sigma)_{\max }<0.001 \\
& \Delta \rho_{\max }=0.42 \mathrm{e} \AA^{-3} \\
& \Delta \rho_{\min }=-0.33 \mathrm{e} \AA^{-3}
\end{aligned}
$$

Special details

Geometry. All e.s.d.'s (except the e.s.d. in the dihedral angle between two 1.s. planes) are estimated using the full covariance matrix. The cell e.s.d.'s are taken into account individually in the estimation of e.s.d.'s in distances, angles and torsion angles; correlations between e.s.d.'s in cell parameters are only used when they are defined by crystal symmetry. An approximate (isotropic) treatment of cell e.s.d.'s is used for estimating e.s.d.'s involving l.s. planes.

Refinement. Refinement of $F^{2}$ against ALL reflections. The weighted $R$-factor $w R$ and goodness of fit $S$ are based on $F^{2}$,

\begin{tabular}{|c|c|c|c|c|}
\hline & $x$ & $y$ & $z$ & $U_{\text {iso }} * / U_{\text {eq }}$ \\
\hline $\mathrm{Cl1}$ & $0.05484(8)$ & $0.40274(8)$ & $0.16648(8)$ & $0.1076(5)$ \\
\hline $\mathrm{Cl} 2$ & $0.19892(9)$ & $0.61061(7)$ & $-0.08078(8)$ & $0.1131(5)$ \\
\hline N1 & $0.26136(16)$ & $0.13047(17)$ & $0.22123(12)$ & $0.0520(6)$ \\
\hline $\mathrm{N} 2$ & $0.32941(18)$ & $0.1240(2)$ & $0.27788(15)$ & $0.0667(7)$ \\
\hline N3 & $0.33128(17)$ & $0.27522(19)$ & $0.21631(14)$ & $0.0597(6)$ \\
\hline $\mathrm{O} 1$ & $-0.01914(15)$ & $0.1842(2)$ & $0.05658(15)$ & $0.0854(8)$ \\
\hline $\mathrm{O} 2$ & 0.09633 (14) & $0.21635(17)$ & $-0.02602(11)$ & $0.0703(6)$ \\
\hline $\mathrm{C} 1$ & $0.0817(4)$ & $0.3050(5)$ & $-0.1491(3)$ & $0.138(2)$ \\
\hline $\mathrm{H} 1 \mathrm{~A}$ & 0.1140 & 0.3576 & -0.1224 & $0.207 *$ \\
\hline H1B & 0.0391 & 0.3323 & -0.1874 & $0.207^{*}$ \\
\hline $\mathrm{H} 1 \mathrm{C}$ & 0.1242 & 0.2634 & -0.1775 & $0.207^{*}$ \\
\hline $\mathrm{C} 2$ & $0.0347(3)$ & $0.2488(4)$ & $-0.0903(2)$ & $0.0953(13)$ \\
\hline $\mathrm{H} 2 \mathrm{~A}$ & 0.0074 & 0.1916 & -0.1164 & $0.114^{*}$ \\
\hline $\mathrm{H} 2 \mathrm{~B}$ & -0.0137 & 0.2883 & -0.0667 & $0.114^{*}$ \\
\hline $\mathrm{C} 3$ & $0.06111(18)$ & $0.19142(19)$ & $0.04424(16)$ & $0.0498(6)$ \\
\hline $\mathrm{C} 4$ & $0.13260(17)$ & 0.17205 (19) & $0.10725(15)$ & $0.0451(6)$ \\
\hline $\mathrm{C} 5$ & $0.13144(18)$ & 0.07882 (19) & $0.14927(16)$ & $0.0494(6)$ \\
\hline C6 & 0.19780 (19) & $0.0596(2)$ & $0.20462(17)$ & $0.0558(7)$ \\
\hline H6 & 0.2000 & -0.0012 & 0.2310 & $0.067^{*}$ \\
\hline $\mathrm{C} 7$ & $0.3669(2)$ & 0.2128 & 0.27135 (19) & $0.0687(9)$ \\
\hline $\mathrm{H} 7$ & 0.4161 & 0.2311 & 0.3038 & $0.082 *$ \\
\hline $\mathrm{C} 8$ & $0.26346(18)$ & 0.22155 (19) & $0.18455(15)$ & $0.0478(6)$ \\
\hline $\mathrm{C} 9$ & $0.19725(16)$ & $0.24312(18)$ & $0.12376(15)$ & $0.0439(6)$ \\
\hline $\mathrm{C} 10$ & $0.20186(17)$ & $0.33997(18)$ & $0.08023(15)$ & $0.0460(6)$ \\
\hline $\mathrm{C} 11$ & $0.1376(2)$ & $0.4141(2)$ & 0.09152 (19) & $0.0597(7)$ \\
\hline $\mathrm{C} 12$ & $0.1379(2)$ & $0.4988(2)$ & $0.0427(2)$ & $0.0717(9)$ \\
\hline H12 & 0.0942 & 0.5477 & 0.0500 & $0.086^{*}$ \\
\hline C13 & $0.2031(2)$ & $0.5087(2)$ & $-0.0156(2)$ & $0.0652(8)$ \\
\hline C14 & $0.2709(2)$ & $0.4403(2)$ & $-0.02570(17)$ & $0.0608(7)$ \\
\hline H14 & 0.3167 & 0.4501 & -0.0641 & $0.073 *$ \\
\hline
\end{tabular}
conventional $R$-factors $R$ are based on $F$, with $F$ set to zero for negative $F^{2}$. The threshold expression of $F^{2}>\sigma\left(F^{2}\right)$ is used only for calculating $R$-factors $(\mathrm{gt})$ etc. and is not relevant to the choice of reflections for refinement. $R$-factors based on $F^{2}$ are statistically about twice as large as those based on $F$, and $R$ - factors based on ALL data will be even larger.

Fractional atomic coordinates and isotropic or equivalent isotropic displacement parameters $\left(\AA^{2}\right)$ 
supporting information

$\begin{array}{lllll}\text { C15 } & 0.26961(18) & 0.3567(2) & 0.02229(16) & 0.0521(7) \\ \text { H15 } & 0.3154 & 0.3099 & 0.0158 & 0.062^{*} \\ \text { C16 } & 0.0598(2) & 0.0018(2) & 0.1339(2) & 0.0667(8) \\ \text { H16A } & 0.0770 & -0.0588 & 0.1604 & 0.100^{*} \\ \text { H16B } & 0.0542 & -0.0093 & 0.0761 & 0.100^{*} \\ \text { H16C } & 0.0026 & 0.0241 & 0.1554 & 0.100^{*}\end{array}$

Atomic displacement parameters $\left(\AA^{2}\right)$

\begin{tabular}{lllllll}
\hline & $U^{11}$ & $U^{22}$ & $U^{33}$ & $U^{12}$ & $U^{13}$ & $U^{23}$ \\
\hline C11 & $0.0972(8)$ & $0.0803(7)$ & $0.1453(10)$ & $0.0092(5)$ & $0.0583(7)$ & $-0.0185(6)$ \\
C12 & $0.1464(10)$ & $0.0607(6)$ & $0.1323(10)$ & $-0.0150(6)$ & $-0.0448(8)$ & $0.0396(6)$ \\
N1 & $0.0569(13)$ & $0.0554(13)$ & $0.0438(11)$ & $0.0063(11)$ & $0.0013(10)$ & $0.0036(10)$ \\
N2 & $0.0705(16)$ & $0.0752(18)$ & $0.0544(14)$ & $0.0091(14)$ & $-0.0085(12)$ & $0.0073(12)$ \\
N3 & $0.0583(14)$ & $0.0654(15)$ & $0.0555(13)$ & $-0.0044(11)$ & $-0.0075(11)$ & $-0.0013(11)$ \\
O1 & $0.0490(13)$ & $0.130(2)$ & $0.0777(15)$ & $-0.0008(13)$ & $0.0030(11)$ & $0.0062(15)$ \\
O2 & $0.0600(12)$ & $0.1014(17)$ & $0.0494(11)$ & $-0.0223(11)$ & $-0.0043(9)$ & $0.0141(10)$ \\
C1 & $0.120(4)$ & $0.192(6)$ & $0.102(3)$ & $-0.071(4)$ & $-0.037(3)$ & $0.068(4)$ \\
C2 & $0.091(3)$ & $0.118(3)$ & $0.077(2)$ & $-0.042(2)$ & $-0.033(2)$ & $0.035(2)$ \\
C3 & $0.0508(16)$ & $0.0464(14)$ & $0.0523(14)$ & $-0.0042(11)$ & $0.0019(12)$ & $-0.0053(11)$ \\
C4 & $0.0476(14)$ & $0.0442(14)$ & $0.0436(13)$ & $0.0007(11)$ & $0.0073(11)$ & $-0.0030(10)$ \\
C5 & $0.0534(15)$ & $0.0442(14)$ & $0.0507(14)$ & $-0.0001(11)$ & $0.0123(12)$ & $-0.0022(11)$ \\
C6 & $0.0678(18)$ & $0.0448(14)$ & $0.0549(15)$ & $0.0036(13)$ & $0.0116(14)$ & $0.0056(12)$ \\
C7 & $0.0627(19)$ & $0.086(2)$ & $0.0576(18)$ & $0.0003(16)$ & $-0.0104(15)$ & $-0.0001(16)$ \\
C8 & $0.0521(15)$ & $0.0480(15)$ & $0.0435(13)$ & $0.0012(11)$ & $0.0037(11)$ & $-0.0013(11)$ \\
C9 & $0.0460(14)$ & $0.0429(13)$ & $0.0428(13)$ & $0.0010(11)$ & $0.0041(10)$ & $-0.0021(10)$ \\
C10 & $0.0474(14)$ & $0.0434(14)$ & $0.0473(14)$ & $-0.0026(11)$ & $-0.0062(11)$ & $-0.0045(11)$ \\
C11 & $0.0558(16)$ & $0.0466(16)$ & $0.0765(19)$ & $0.0008(12)$ & $0.0009(14)$ & $-0.0113(13)$ \\
C12 & $0.067(2)$ & $0.0407(16)$ & $0.107(3)$ & $0.0075(13)$ & $-0.0178(19)$ & $-0.0072(16)$ \\
C13 & $0.077(2)$ & $0.0452(16)$ & $0.074(2)$ & $-0.0100(15)$ & $-0.0236(17)$ & $0.0054(14)$ \\
C14 & $0.0716(19)$ & $0.0550(17)$ & $0.0559(16)$ & $-0.0131(14)$ & $-0.0018(14)$ & $0.0030(13)$ \\
C15 & $0.0542(16)$ & $0.0476(15)$ & $0.0545(15)$ & $-0.0030(12)$ & $-0.0005(12)$ & $0.0026(12)$ \\
C16 & $0.0708(19)$ & $0.0487(16)$ & $0.081(2)$ & $-0.0097(14)$ & $0.0069(16)$ & $-0.0006(15)$ \\
& & & & & & \\
\hline
\end{tabular}

Geometric parameters $\left(\AA,{ }^{\circ}\right)$

\begin{tabular}{llll}
\hline $\mathrm{C} 11-\mathrm{C} 11$ & $1.733(3)$ & $\mathrm{C} 4-\mathrm{C} 5$ & $1.437(4)$ \\
$\mathrm{C} 12-\mathrm{C} 13$ & $1.743(3)$ & $\mathrm{C} 5-\mathrm{C} 6$ & $1.355(4)$ \\
$\mathrm{N} 1-\mathrm{C} 6$ & $1.365(4)$ & $\mathrm{C} 5-\mathrm{C} 16$ & $1.502(4)$ \\
$\mathrm{N} 1-\mathrm{N} 2$ & $1.366(3)$ & $\mathrm{C} 6-\mathrm{H} 6$ & 0.9300 \\
$\mathrm{~N} 1-\mathrm{C} 8$ & $1.371(3)$ & $\mathrm{C} 7-\mathrm{H} 7$ & 0.9300 \\
$\mathrm{~N} 2-\mathrm{C} 7$ & $1.326(4)$ & $\mathrm{C} 8-\mathrm{C} 9$ & $1.421(4)$ \\
$\mathrm{N} 3-\mathrm{C} 8$ & $1.338(3)$ & $\mathrm{C} 9-\mathrm{C} 10$ & $1.493(4)$ \\
$\mathrm{N} 3-\mathrm{C} 7$ & $1.341(4)$ & $\mathrm{C} 10-\mathrm{C} 11$ & $1.389(4)$ \\
$\mathrm{O} 1-\mathrm{C} 3$ & $1.200(3)$ & $\mathrm{C} 10-\mathrm{C} 15$ & $1.393(4)$ \\
$\mathrm{O} 2-\mathrm{C} 3$ & $1.304(3)$ & $\mathrm{C} 11-\mathrm{C} 12$ & $1.398(4)$ \\
$\mathrm{O} 2-\mathrm{C} 2$ & $1.455(4)$ & $\mathrm{C} 12-\mathrm{C} 13$ & $1.357(5)$ \\
$\mathrm{C} 1-\mathrm{C} 2$ & $1.406(5)$ & $\mathrm{C} 12-\mathrm{H} 12$ & 0.9300
\end{tabular}




\begin{tabular}{|c|c|c|c|}
\hline $\mathrm{C} 1-\mathrm{H} 1 \mathrm{~A}$ & 0.9600 & $\mathrm{C} 13-\mathrm{C} 14$ & $1.371(4)$ \\
\hline $\mathrm{C} 1-\mathrm{H} 1 \mathrm{~B}$ & 0.9600 & $\mathrm{C} 14-\mathrm{C} 15$ & $1.376(4)$ \\
\hline $\mathrm{C} 1-\mathrm{H} 1 \mathrm{C}$ & 0.9600 & C14-H14 & 0.9300 \\
\hline $\mathrm{C} 2-\mathrm{H} 2 \mathrm{~A}$ & 0.9700 & C15-H15 & 0.9300 \\
\hline $\mathrm{C} 2-\mathrm{H} 2 \mathrm{~B}$ & 0.9700 & $\mathrm{C} 16-\mathrm{H} 16 \mathrm{~A}$ & 0.9600 \\
\hline $\mathrm{C} 3-\mathrm{C} 4$ & $1.494(4)$ & $\mathrm{C} 16-\mathrm{H} 16 \mathrm{~B}$ & 0.9600 \\
\hline $\mathrm{C} 4-\mathrm{C} 9$ & $1.378(4)$ & $\mathrm{C} 16-\mathrm{H} 16 \mathrm{C}$ & 0.9600 \\
\hline $\mathrm{C} 6-\mathrm{N} 1-\mathrm{N} 2$ & $126.2(2)$ & $\mathrm{N} 3-\mathrm{C} 7-\mathrm{H} 7$ & 121.2 \\
\hline $\mathrm{C} 6-\mathrm{N} 1-\mathrm{C} 8$ & $124.0(2)$ & $\mathrm{N} 3-\mathrm{C} 8-\mathrm{N} 1$ & $109.6(2)$ \\
\hline $\mathrm{N} 2-\mathrm{N} 1-\mathrm{C} 8$ & $109.7(2)$ & $\mathrm{N} 3-\mathrm{C} 8-\mathrm{C} 9$ & $132.1(2)$ \\
\hline $\mathrm{C} 7-\mathrm{N} 2-\mathrm{N} 1$ & $101.1(2)$ & $\mathrm{N} 1-\mathrm{C} 8-\mathrm{C} 9$ & $118.4(2)$ \\
\hline $\mathrm{C} 8-\mathrm{N} 3-\mathrm{C} 7$ & $102.1(3)$ & $\mathrm{C} 4-\mathrm{C} 9-\mathrm{C} 8$ & $117.7(2)$ \\
\hline $\mathrm{C} 3-\mathrm{O} 2-\mathrm{C} 2$ & $117.9(2)$ & $\mathrm{C} 4-\mathrm{C} 9-\mathrm{C} 10$ & $123.4(2)$ \\
\hline $\mathrm{C} 2-\mathrm{C} 1-\mathrm{H} 1 \mathrm{~A}$ & 109.5 & $\mathrm{C} 8-\mathrm{C} 9-\mathrm{C} 10$ & 118.9 (2) \\
\hline $\mathrm{C} 2-\mathrm{C} 1-\mathrm{H} 1 \mathrm{~B}$ & 109.5 & $\mathrm{C} 11-\mathrm{C} 10-\mathrm{C} 15$ & $117.3(3)$ \\
\hline $\mathrm{H} 1 \mathrm{~A}-\mathrm{C} 1-\mathrm{H} 1 \mathrm{~B}$ & 109.5 & $\mathrm{C} 11-\mathrm{C} 10-\mathrm{C} 9$ & $122.6(2)$ \\
\hline $\mathrm{C} 2-\mathrm{C} 1-\mathrm{H} 1 \mathrm{C}$ & 109.5 & $\mathrm{C} 15-\mathrm{C} 10-\mathrm{C} 9$ & $120.0(2)$ \\
\hline $\mathrm{H} 1 \mathrm{~A}-\mathrm{C} 1-\mathrm{H} 1 \mathrm{C}$ & 109.5 & $\mathrm{C} 10-\mathrm{C} 11-\mathrm{C} 12$ & $120.9(3)$ \\
\hline $\mathrm{H} 1 \mathrm{~B}-\mathrm{C} 1-\mathrm{H} 1 \mathrm{C}$ & 109.5 & $\mathrm{C} 10-\mathrm{C} 11-\mathrm{C} 11$ & $120.5(2)$ \\
\hline $\mathrm{C} 1-\mathrm{C} 2-\mathrm{O} 2$ & $110.6(3)$ & $\mathrm{C} 12-\mathrm{C} 11-\mathrm{Cl} 1$ & $118.6(2)$ \\
\hline $\mathrm{C} 1-\mathrm{C} 2-\mathrm{H} 2 \mathrm{~A}$ & 109.5 & $\mathrm{C} 13-\mathrm{C} 12-\mathrm{C} 11$ & $119.0(3)$ \\
\hline $\mathrm{O} 2-\mathrm{C} 2-\mathrm{H} 2 \mathrm{~A}$ & 109.5 & $\mathrm{C} 13-\mathrm{C} 12-\mathrm{H} 12$ & 120.5 \\
\hline $\mathrm{C} 1-\mathrm{C} 2-\mathrm{H} 2 \mathrm{~B}$ & 109.5 & $\mathrm{C} 11-\mathrm{C} 12-\mathrm{H} 12$ & 120.5 \\
\hline $\mathrm{O} 2-\mathrm{C} 2-\mathrm{H} 2 \mathrm{~B}$ & 109.5 & $\mathrm{C} 12-\mathrm{C} 13-\mathrm{C} 14$ & $122.0(3)$ \\
\hline $\mathrm{H} 2 \mathrm{~A}-\mathrm{C} 2-\mathrm{H} 2 \mathrm{~B}$ & 108.1 & $\mathrm{C} 12-\mathrm{C} 13-\mathrm{Cl} 2$ & $118.9(3)$ \\
\hline $\mathrm{O} 1-\mathrm{C} 3-\mathrm{O} 2$ & $124.0(3)$ & $\mathrm{C} 14-\mathrm{C} 13-\mathrm{Cl} 2$ & $119.1(3)$ \\
\hline $\mathrm{O} 1-\mathrm{C} 3-\mathrm{C} 4$ & $124.1(3)$ & $\mathrm{C} 13-\mathrm{C} 14-\mathrm{C} 15$ & $118.5(3)$ \\
\hline $\mathrm{O} 2-\mathrm{C} 3-\mathrm{C} 4$ & $111.9(2)$ & $\mathrm{C} 13-\mathrm{C} 14-\mathrm{H} 14$ & 120.8 \\
\hline $\mathrm{C} 9-\mathrm{C} 4-\mathrm{C} 5$ & $121.8(2)$ & $\mathrm{C} 15-\mathrm{C} 14-\mathrm{H} 14$ & 120.8 \\
\hline $\mathrm{C} 9-\mathrm{C} 4-\mathrm{C} 3$ & $119.8(2)$ & $\mathrm{C} 14-\mathrm{C} 15-\mathrm{C} 10$ & $122.1(3)$ \\
\hline $\mathrm{C} 5-\mathrm{C} 4-\mathrm{C} 3$ & $118.4(2)$ & $\mathrm{C} 14-\mathrm{C} 15-\mathrm{H} 15$ & 119.0 \\
\hline $\mathrm{C} 6-\mathrm{C} 5-\mathrm{C} 4$ & $118.6(2)$ & $\mathrm{C} 10-\mathrm{C} 15-\mathrm{H} 15$ & 119.0 \\
\hline $\mathrm{C} 6-\mathrm{C} 5-\mathrm{C} 16$ & $118.8(3)$ & $\mathrm{C} 5-\mathrm{C} 16-\mathrm{H} 16 \mathrm{~A}$ & 109.5 \\
\hline $\mathrm{C} 4-\mathrm{C} 5-\mathrm{C} 16$ & $122.5(3)$ & $\mathrm{C} 5-\mathrm{C} 16-\mathrm{H} 16 \mathrm{~B}$ & 109.5 \\
\hline $\mathrm{C} 5-\mathrm{C} 6-\mathrm{N} 1$ & $119.4(2)$ & $\mathrm{H} 16 \mathrm{~A}-\mathrm{C} 16-\mathrm{H} 16 \mathrm{~B}$ & 109.5 \\
\hline $\mathrm{C} 5-\mathrm{C} 6-\mathrm{H} 6$ & 120.3 & $\mathrm{C} 5-\mathrm{C} 16-\mathrm{H} 16 \mathrm{C}$ & 109.5 \\
\hline $\mathrm{N} 1-\mathrm{C} 6-\mathrm{H} 6$ & 120.3 & $\mathrm{H} 16 \mathrm{~A}-\mathrm{C} 16-\mathrm{H} 16 \mathrm{C}$ & 109.5 \\
\hline $\mathrm{N} 2-\mathrm{C} 7-\mathrm{N} 3$ & $117.6(3)$ & $\mathrm{H} 16 \mathrm{~B}-\mathrm{C} 16-\mathrm{H} 16 \mathrm{C}$ & 109.5 \\
\hline $\mathrm{N} 2-\mathrm{C} 7-\mathrm{H} 7$ & 121.2 & & \\
\hline
\end{tabular}

Hydrogen-bond geometry $\left(\AA,{ }^{\circ}\right)$

\begin{tabular}{lllll}
\hline$D-\mathrm{H} \cdots A$ & $D-\mathrm{H}$ & $\mathrm{H} \cdots A$ & $D \cdots A$ & $D-\mathrm{H} \cdots A$ \\
\hline $\mathrm{C} 7-\mathrm{H} 7 \cdots \mathrm{O}^{\mathrm{i}}$ & 0.93 & 2.55 & $3.296(4)$ & 137 \\
\hline
\end{tabular}

Symmetry code: (i) $x+1 / 2, y,-z+1 / 2$. 